\title{
Hematoquecia letal por angiostrongilosis abdominal
}

\begin{abstract}
Gerzaín Rodríguez
Laboratorio de Patología, Instituto Nacional de Salud; Departamento de Patología, Facultad de Medicina, Universidad Nacional de Colombia, Santa Fe de Bogotá, D.C., Colombia

Una niña indígena de 4 años de edad, procedente de Carurú, Vaupés, un municipio semiselvático de 2.000 habitantes, presentó hematoquecia repetida con síncope y muerte 4 días después, a pesar de recibir transfusiones sanguíneas. El estudio postmortem descubrió dos masas de 3-4 cm de diámetro en el íleon distal que, al microscopio, correspondieron a inflamación severa de toda la pared intestinal, rica en eosinófilos y en granulomas con células gigantes que circunscribían y fagocitaban huevos y larvas de Angiostrongylus costaricensis, helminto situado también dentro de las arterias de la submucosa, vasos que presentaban engrosamiento de la íntima y focos con inflamación granulomatosa con eosinófilos. La niña no presentó leucocitosis ni eosinofilia.

Este es el segundo caso de angiostrongilosis de esta región. El primero fue el de una mujer indígena de 34 años también con hematoquecia anemizante. La angiostrongilosis abdominal se diagnosticó por primera vez en Colombia en 1979, en un niño de 5 años, de Dagua, Valle. La niña aquí presentada es el sexto paciente con esta entidad en Colombia. La hematoquecia como manifestación predominante de la angiostrongilosis es extraordinaria para una enfermedad parasitaria; se ha registrado solamente en tres ocasiones anteriores. La abundancia de cristales de Charcot-Leyden, que se tiñen muy bien con la coloración de ZiehlNeelsen o de Fite-Faraco, en los infiltrados de la pared intestinal, sugiere que su búsqueda en las heces puede ser una ayuda en el diagnóstico de casos sospechosos, pues, los huevos y las larvas no se eliminan en las heces y el diagnóstico definitivo de la angiostrongilosis es histopatológico. Revisamos las características clínicas, parasitológicas e histopatológicas de esta parasitosis que será más prevalente en Colombia a medida que los médicos, especialmente los patólogos, la conozcan mejor.
\end{abstract}

Palabras clave: angiostrongilosis abdominal, parasitismo intestinal, hematoquecia, cristales de Charcot-Leyden, vasculitis eosinofilica.

\section{Lethal hematochezia due to abdominal angiostrongylosis}

A four-year old native indian girl from Carurú (Vaupés), a sylvatic area of 2,000 inhabitants, near the Colombian border with Brazil, presented with relapsing hematochezia and syncope and died four days later in Mitú $\left(70^{\circ}, 10^{\prime}, 24^{\prime \prime}\right.$ WL; $\left.01^{\circ}, 11^{\prime}, 54^{\prime \prime} \mathrm{NL}\right)$, despite of several blood transfusions. The blood count showed neither leukocytosis nor eosinophilia. The postmortem examination revealed two masses of 3-4 cm in diameter, located at the distal ileum which on light microscopy showed a severe inflammation of the whole intestinal wall, rich in eosinophils, and giant cell granulomas surrounding and phagocytizing eggs and larvae of Angystrongylus costaricensis, which was also seen in the submucosal arteries, vessels that displayed thickening of the intima and granulomatous inflammation foci with eosinophilia.

This is the second angiostrongylosis case from this region; the first one was in a 34-year old native indian woman also with anemia due to hematochezia. Abdominal angiostrongylosis was diagnosed for the first time in Colombia in 1979 in a 5-year old boy from Dagua (Valle). Our case is the sixth patient diagnosed with this disease in Colombia. Hematochezia as a predominant manifestation of angiostrongylosis rarely occurs. It has been previously reported only on three occassions. 
The great number of eosinophilic Charcot-Leyden crystals present in the intestinal wall infiltrates also stains very well either with the Ziehl-Neelsen or Fite-Faraco stains; we suggest that its search in fecal smears might be of great aid in the diagnosis of suspected cases, since $A$. costaricensis eggs and larvae are not eliminated in human feces and, consequently, its final diagnosis is frequently histopathological. We reviewed the clinical, parasitological and histopathological characteristics of this parasitosis whose prevalence will probably increase in Colombia as physicians, specially pathologists, get to know it better.

Key words: abdominal angiostrongylosis, intestinal parasitosis, hematochezia, CharcotLeyden crystals, eosinophilic vasculitis.

El hombre sólo ve lo que conoce, Goethe

A medida que más áreas de la geografía colombiana disponen de personal de salud, aparecen enfermedades consideradas como raras o exóticas. El apoyo que el Laboratorio de Patología del Instituto Nacional de Salud ha brindado durante años a los departamentos sin este servicio, ha permitido que los médicos de las áreas rurales o semiselváticas dispongan de diagnósticos más precisos de las enfermedades de sus pacientes. También, los aspectos benéficos de las actuales leyes de la salud facilitan que los pacientes de estas zonas marginadas $u$ olvidadas puedan tener atención médica en los grandes centros hospitalarios de las capitales, lo cual era otrora poco menos que una utopía. Dos ejemplos de enfermedades importantes que no se hubieran detectado sin la asociación entre el INS y los médicos de estas zonas geográficas son el pénfigo foliáceo en indígenas (1) y la lobomicosis $(2,3)$. En este trabajo, presentamos otra observación única, originada en el interés por sus pacientes de los médicos y otros funcionarios de la salud de Carurú y Mitú, Vaupés.

\section{Descripción del caso}

Una niña indigena tayuca, natural de Bacatí, Vaupés, procedente de la vereda El Carmen del municipio de Carurú, Vaupés, fue llevada al Hospital de Mitú, por presentar deposiciones con sangre. La información general se obtuvo de su padre, con quien la comunicación no era fácil porque no habla bien el español.

\footnotetext{
Correspondencia:

Laboratorio de Patologia, Instituto Nacional de Saiud, Apartado aéreo 80334, Santa Fe de Bogotá, D.C., Colombia. grodríguez@hemagogus.ins.gov.co

Recibido: 03/01/00; aceptado: 24/03/00
}

Informó que la niña estaba enferma desde hacía dos días cuando comenzó a presentar anorexia, dolor abdominal moderado y vómito postprandial escaso, seguido de diarrea discreta y de dos deposiciones con sangre y abundantes coágulos, con síncope posterior. La llevó al puesto de salud de Carurú en donde el médico y el enfermero constataron anemia aguda y le hicieron una transfusión de sangre del padre, sin clasificación del grupo sanguíneo por no disponer de este procedimiento. No le encontraron masas abdominales ni alteraciones en el tacto rectal, el cual fue un poco doloroso y dejó el guante impregnado de sangre.

Por la gravedad del cuadro clínico, la remitieron a Mitú, en donde se describió una niña con palidez mucocutánea generalizada y en condiciones generales aceptables, con frecuencias cardiaca de $120 / \mathrm{min}$, frecuencia respiratoria de $24 / \mathrm{min}$, temperatura de $36^{\circ} \mathrm{C}$ y peso de $12 \mathrm{~kg}$; cuello, boca y extremidades, normales. La auscultación no reveló soplos ni sibilancias. El abdomen se presentaba blando, depresible, sin dolor a la palpación, con defensa abdominal voluntaria; no se palpaban masas ni visceromegalias, aunque en otro examen se detectó una masa en hipocondrio derecho, de $2 \times 1 \mathrm{~cm}$, que no parecía ser visceral sino de los planos musculares profundos. Había peristaltismo y se confirmó el sangrado rectal con síndrome anémico agudo. Se sugirieron las posibilidades de gastritis erosiva aguda, úlcera gástrica o duodenal y poliparasitismo intestinal.

\section{Laboratorio}

Los exámenes de laboratorio dieron los siguientes resultados: hemoglobina, $6 \mathrm{~g} \%$; hematocrito, $18 \%$; leucocitos, $9.000 / \mathrm{mm}^{3}$; linfocitos, $24 \%$; polimorfonucleares, $74 \%$; eosinófilos, $2 \%$; plaquetas, $200.000 / \mathrm{mm}^{3}$; tiempo de protrombina, $18 \mathrm{seg}$; microcitos, ++; hipocromía, ++; grupo 
sanguíneo, O Rh+; hemoparásitos: positivo para Plasmodium vivax. El examen coprológico arrojó estos resultados: hematest, ++; leucocitos: $>5$ por campo; moco, ++; parásitos: negativo. El parcial de orina dió estos resultados: densidad: 1.010; acetona, $15 \mathrm{mg} / \mathrm{dl}$; leucocitos, 2-3/campo; hematíes, 1-2/campo; moco, ++; bacterias, +.

Se ordenó lactato de Ringer, vitamina K, ranitidina, sonda nasogástrica y la administración de oxígeno. Visto el resultado de los hemoparásitos, se prescribió cloroquina, $300 \mathrm{mg}$ de dosis total, con una dosis inicial de $120 \mathrm{mg}$ y $90 \mathrm{mg}$ cada 24 horas y primaquina, $3,6 \mathrm{mg} /$ día durante 14 días. En las primeras 24 horas de permanencia en el hospital, hizo una deposición sanguinolenta de 500 $\mathrm{ml}$ aproximadamente, por lo cual se transfundio con $150 \mathrm{ml}$ de sangre. El cuadro hemático antes de la transfusión mostró: hemoglobina, $5 \mathrm{~g} \%$; hematocrito: $15 \%$; leucocitos $10.100 / \mathrm{m}^{3}$ : polimorfonucleares, $56 \%$; linfocitos $38 \%$; eosinófilos: $1 \%$; basófilos: $1 \%$; cayados $4 \%$.

La niña siguió presentando fiebre $\left(37,7^{\circ} \mathrm{C}\right)$, rectorragia leve, álgida, irritable, quejumbrosa y falleció al segundo día de hospitalizada, pese a las maniobras de reanimación, la intubación orotraqueal y la aplicación de adrenalina y atropina.

\section{Hallazgos de autopsia}

Los médicos de Mitú realizaron una autopsia parcial, con exploración de la cavidad abdominal, en la cual encontraron dos masas nodulares de 3 $\mathrm{cm}$ de diámetro, aproximadamente, que hacían parte de la pared intestinal del íleon distal (figura 1).

Su estudio histopatológico, realizado en el INS, se ilustra en las figuras $2-12$ y se resume así: engrosamiento severo de todas las capas de la pared yeyunal debido a un proceso inflamatorio granulomatoso, rico en eosinófilos, con presencia de huevos y larvas de un helminto en diferentes fases de desarrollo (figuras 2-4).

Los granulomas son epitelioides y de células gigantes de tipo cuerpo extraño, que fagocitan los huevos o las larvas, los cuales, en general, están circunscritos por los granulomas (figuras 6-10).

Los eosinófilos predominan en amplias áreas desde la lámina propria de la mucosa hasta la serosa y el peritoneo; forman abundantes cristales de

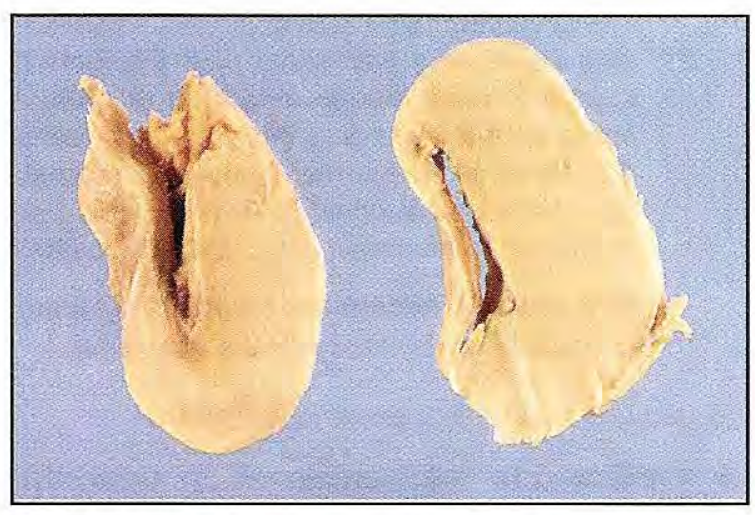

Figura 1. Corte de un segmento del íleon distal con engrosamiento homogéneo y amarillento de la pared y notoria disminución de la luz intestinal.

Charcot-Leyden, eosinófilos con la coloración de hematoxilina-eosina y claramente demostrables como ácido-alcohol resistentes con la coloración de Ziehl-Neelsen o de Fite-Faraco (figuras 4-7, $9,11)$.Se observan, además, abundantes plasmocitos, linfocitos y áreas de fibrosis.

En varios cortes se demuestran helmintos adultos dentro de la luz de arterias intestinales de la submucosa (figuras 2, 3 y 5). En cortes seriados, se puede ver la proliferación de la íntima de pocos de estos vasos, la ruptura de la lámina elástica interna y la oclusión final del vaso, así como la infiltración de su pared por células inflamatorias, principalmente eosinófilos; también se ven granulomas intraarteriales que fagocitan componentes del parásito o sus huevos; el cuadro es de una arteritis eosinofílica (figura 5).

Los huevos del parásito tienen diversas fases de desarrollo, lo cual se traduce en aspectos morfológicos distintos. Miden entre 40-70 x 20$42 \mu \mathrm{m}$ y aparecen como masas eosinófilas vacuoladas u homogéneas, sin núcleos o como estadios de mórulas con células claras y con profusión de núcleos (figuras 6-9). Están cubiertos por una cutícula muy delgada, casi inaparente. Usualmente, están rodeados por un espacio claro amplio que puede resultar de la retracción de sus células durante el proceso del tejido o de su localización dentro de pequeños vasos sanguíneos. Es frecuente verlos rodeados por los granulomas o fagocitados por las células gigantes. Gradualmente, la multiplicación del embrión 


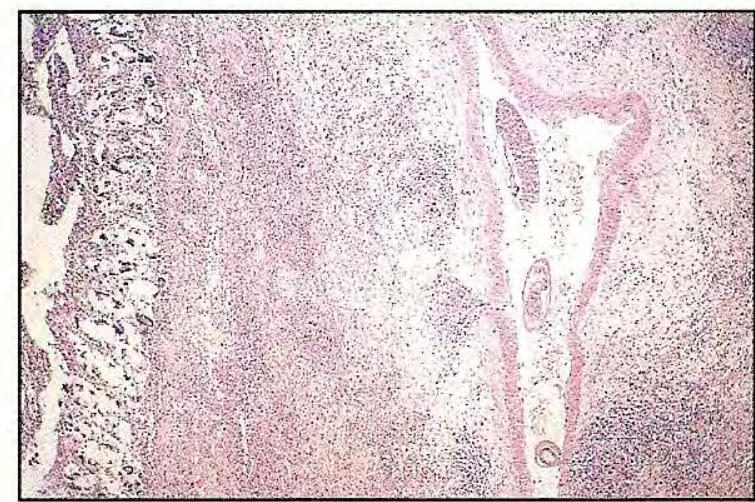

Figura 2. Imagen microscópica panorámica; hay autólisis de la mucosa, engrosamiento notorio de la submucosa por inflamación difusa y una arteria con 3 parásitos adultos en la luz, HE, 4X.

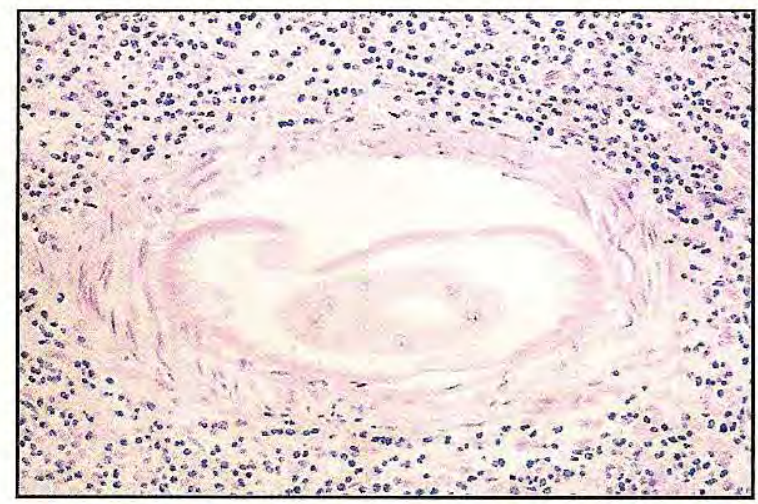

Figura 4. Este parásito ocupa casi toda la luz arterial; el infiltrado inflamatorio vecino, difuso, es muy rico en eosinófilos, HE, 25X.

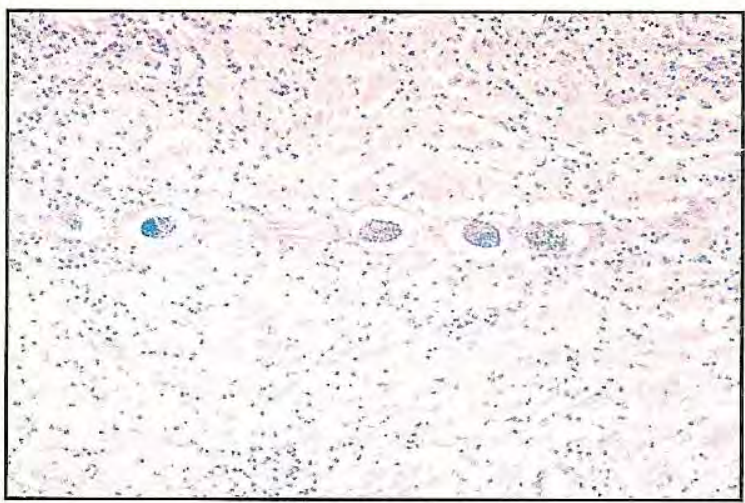

Figura 6. Huevos del parásito en fila india, que revela su localización en vasos diminutos; están rodeados de inflamación difusa rica en eosinófilos, HE, 15X.

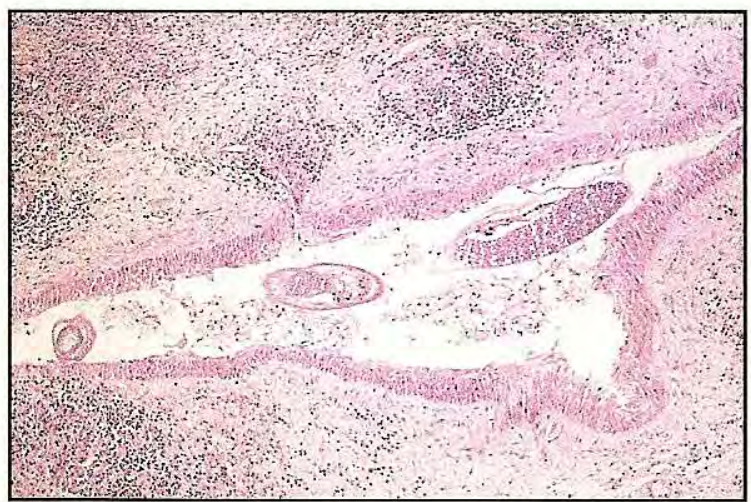

Figura 3. A mayor aumento, se ve la cutícula de los helmintos y algunos de sus componentes celulares. La pared arterial no tiene mayor lesión, pero, en algunos focos, los infiltrados inflamatorios tocan su pared muscular, $\mathrm{HE}, 6,3 \mathrm{X}$.

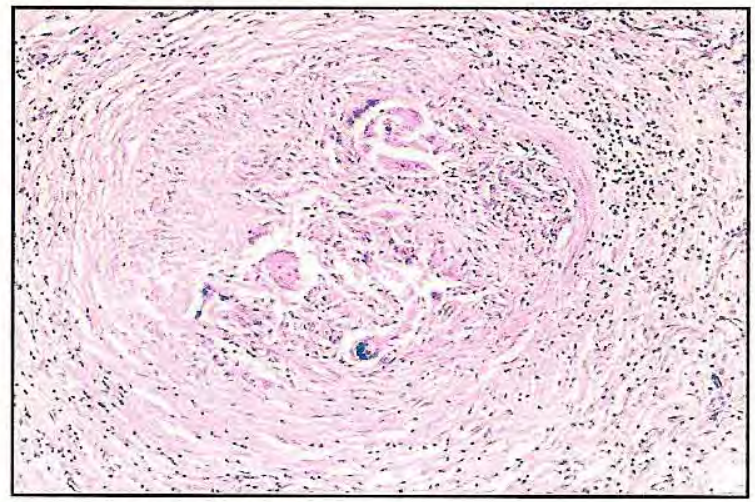

Figura 5. Arteria de pared gruesa y luz ocluida por inflamación granulomatosa con eosinófilos, con algunos fragmentos parasitarios, $\mathrm{HE}, 12,5 \mathrm{X}$.

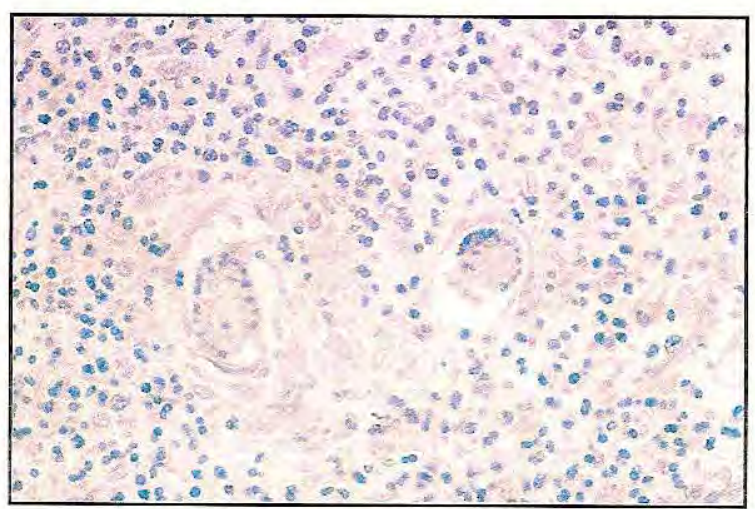

Figura 7. Huevos del parásito en segmentación moruloide, rodeados de macrófagos, linfocitos, plasmocitos, eosinófilos y cristales de Charcot-Leyden, poco aparentes en esta coloración, HE, 40X. 


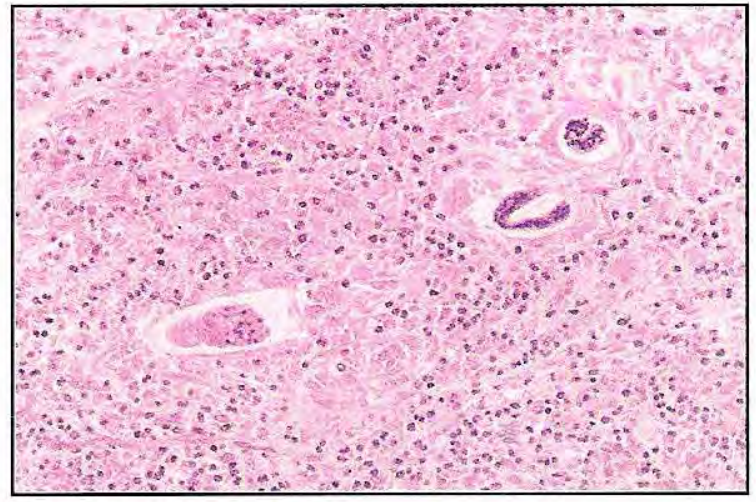

Figura 8. Huevos de $A$. costaricensis originando larvas, rodeados de severa inflamación, HE, 25X.

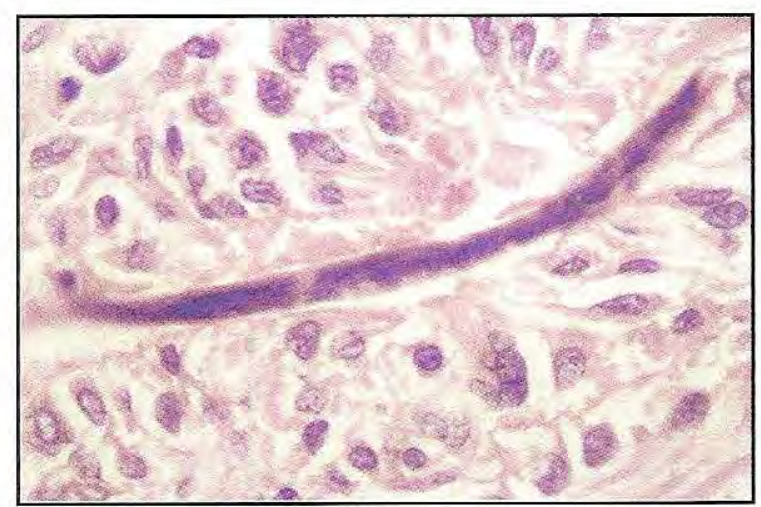

Figura 10. Larva rodeada por un granuloma de macrófagos epitelioides, HE, 40X.

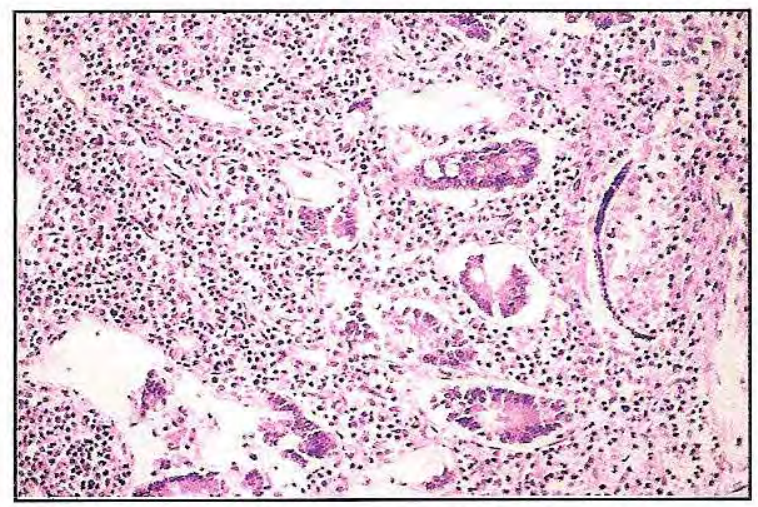

Figura 12. Esta larva se encuentra en la lámina propria de la mucosa,una localización poco frecuente de $A$. costaricensis, a diferencia de las larvas de Strongyloides que son más abundantes en esta zona. En la parte superior, las estructuras más prominentes y basófilas son fragmentos de glándulas mucosas con autolisis, HE, 16X.

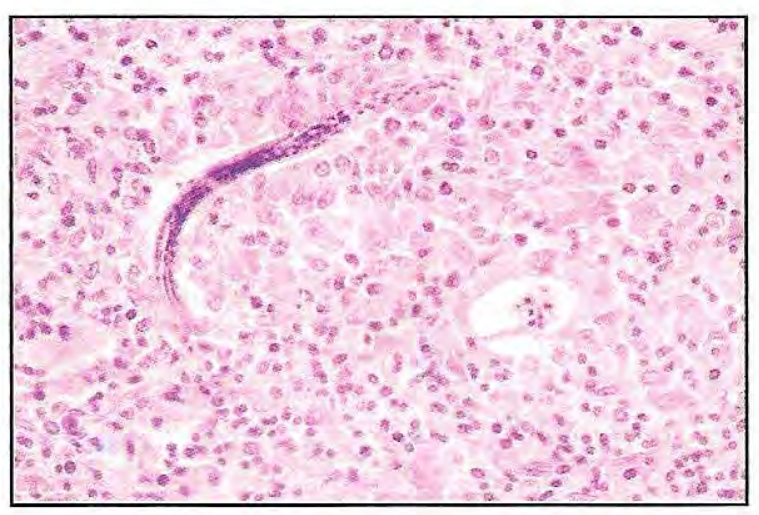

Figura 9. Huevo y larva de primer estadio en la submucosa, rodeados de macrófagos, linfocitos y eosinófilos, HE, 40X.

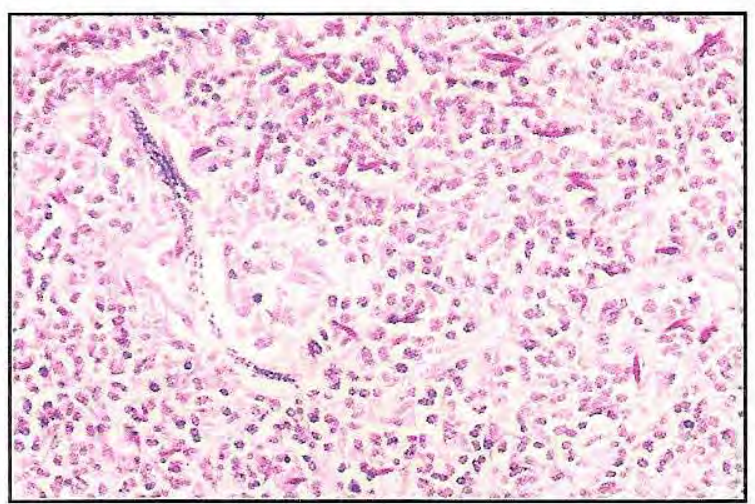

Figura 11. Larva rodeada de inflamación rica en eosinófilos; los cristales de Charcot-Leyden resaltan por su abundancia y ácido-alcohol resistencia, FF, 30X.

conforma una larva muy rica en células y de aspecto recto o entorchado, de 6 a $8 \mu \mathrm{m}$ de diámetro y longitud máxima de $192 \mu$ m, también rodeada o en destrucción por los granulomas y los eosinófilos del infiltrado (figuras 6-12).

El diagnóstico histopatológico es el de angiostrongilosis o parastrongilosis abdominal.

\section{Discusión}

La angiostrongilosis abdominal fue descrita en Costa Rica desde los años 50 y allí mismo se caracterizó como una nueva parasitosis por Morera y Céspedes entre 1967-71 (4,5). Más del $95 \%$ de los afectados son niños menores de 12 años de edad $(6,7)$, aunque la entidad también ocurre en adultos. En Costa Rica, se considera un problema de salud pública con mayor importancia 
que la tuberculosis infantil, con una prevalencia anual cercana a 12/100.000 habitantes (8).

La enfermedad ocurre en Centroamérica, Suramérica y en algunas islas del Caribe, como Martinica y Guadalupe (9) y hay un sólo caso descrito en Africa, en Zaire (10). En Estados Unidos, se ha descrito en roedores (11) y en pacientes procedentes de Puerto Rico y República Dominicana (12-14). En Brasil, hasta 1995, se habían informado cerca de 50 casos (15-18). Los pacientes colombianos los analizaremos más adelante.

Es posible que la enfermedad pase sin diagnóstico por falta de conocimiento de la misma, ya que las condiciones epidemiológicas de Costa Rica no son significativamente diferentes del resto de Latinoamérica (8).

La enfermedad es producida por un nemátodo que ha recibido varios nombres, tales como Angiostrongylus y Morerastrongylus costaricensis (19), cuya nomenclatura actualmente recomendada es Parastrongylus costaricensis, por prioridades en nomenclatura y por la morfología de la bolsa copuladora del macho $(20,21)$. Así mismo, se sugiere denominar la enfermedad como parastrongilosis (22).

El parásito vive en las arterias mesentéricas que irrigan la región ileocecal de varias especies de roedores, tales como Sigmodon hyspidus (rata algodonera) y Rattus rattus (rata común) $(19,23)$, vasos en los cuales pueden demostrarse con facilidad, pues la hembra tiene $32 \mathrm{~mm}$ de longitud $x 0,5 \mathrm{~mm}$ de anchura y el macho $20 \mathrm{~mm} \times 0,5$ $\mathrm{mm}(19,23,24)$. Sus huevos son llevados por el torrente sanguíneo a los capilares de la mucosa y de ellos salen larvas de primer estadio que atraviesan la mucosa intestinal y son eliminadas en las heces del roedor. Las larvas se identifican con facilidad en los cortes histológicos de la mucosa del ciego; no desencadenan mayor reacción inflamatoria en este huésped (23).

En el medio ambiente son ingeridas por varias especies de moluscos (17), siendo el más frecuente Vaginulus plebeius $(7,19,24), 50 \%$ de los cuales, en promedio, están infectados con el parásito en Costa Rica (24). En estas babosas, las larvas perforan la pared intestinal y migran hasta localizarse en tejidos superficiales, alcanzando, después de dos mudas, su madurez y capacidad infectante a los 18 días de la infección del molusco (24). En la babosa, pueden permanecer viables durante meses o salen eliminadas con la secreción mucosa que cubre la piel del molusco (23). La babosa, con las larvas infectantes de tercer estadio, es ingerida de nuevo por el roedor; en el íleon distal o en el ciego atraviesan la pared e invaden los vasos linfáticos, sufren una nueva muda y en cerca de 10 días penetran a las arterias ileocecales que son su hábitat definitivo. Los parásitos adultos comienzan a producir huevos a los 18 días de ingeridas las larvas y a los 24 días ya se las puede detectar en las heces del roedor (24).

Es posible que las larvas migren de la babosa y contaminen legumbres $u$ otros alimentos humanos, o que los niños ingieran las larvas accidentalmente o por jugar con las babosas (7). Los niños tienden a realizar estas prácticas con mayor frecuencia que las niñas, por lo cual ellos son los más frecuentemente afectados (6,7). La ingestión de ensaladas contaminadas con las larvas se postula como la más importante fuente de contaminación al humano. La larva penetra la mucosa intestinal y sigue el mismo recorrido que se describió antes para los roedores. Los huevos son acarreados por el torrente sanguíneo a la mucosa y a la submucosa y comienzan su transformación en larvas de primer estadio, las cuales no son capaces de perforar la mucosa y de penetrar en la luz intestinal. Es decir, en el hombre no se eliminan los huevos ni las larvas en la materia fecal. La acumulación progresiva de huevos y de larvas origina una severa inflamación con eosinófilos y granulomas a cuerpo extraño que termina por comprometer todas las capas intestinales, que se tornan gruesas, empastadas con formación de masa que reduce la luz intestinal; todo esto, junto con la arteritis eosinofílica y granulomatosa por la presencia de los vermes adultos en las arterias, produce un cuadro sintomático amplio.

La clínica se deriva del daño severo de la pared intestinal e incluye dolor abdominal, fiebre, anorexia, vómito, diarrea o constipación y pérdida de peso, entre otros $(6,7,17)$. La rigidez abdominal, 
la sensibilidad, la palpación de masa en la fosa ilíaca derecha y el dolor al tacto rectal hacen que se diagnostique apendicitis aguda $y$, a veces, un tumor abdominal. La presencia de un abdomen agudo exige una conducta quirúrgica.

La hematoquecia o hemorragia rectal de sangre fresca es ocasional en la angiostrongilosis abdominal. Hay tres observaciones previas de esta presentación: una en un hombre dominicano de 41 años residente en Nueva York, con varios sangrados rectales (12); otra en un niño de 5 años de Guadalupe (9) y la tercera en una mujer de 34 años, natural y procedente de Mitú, atendida allí y en el Hospital San Juan de Dios de Bogotá (25). La cuarta observación es la niña presentada en este trabajo, cuyo sangrado rectal masivo la llevó a la muerte por anemia aguda.

El laboratorio muestra leucocitosis, a veces muy alta, entre 20-30.000 leucocitos $/ \mathrm{mm}^{3}$, con eosinofilia hasta de $60 \%$, o aún mayor, en el $75 \%$ de los casos $(6,7)$. Ninguno de estos criterios estaba presente en la niña, ni en otros 2 pacientes con hematoquecia, pero sí estaban presentes en el niño de Guadalupe (9), por lo cual la ausencia de leucocitosis y de eosinofilia no se puede atribuir con certeza a la anemia aguda severa que en todos estos casos hallegado a estar entre 4$6 \mathrm{~g} \%$ de hemoglobina.

Los rayos $\mathrm{X}$ son útiles, especialmente el colon por enema que revela cambios en el llenado y aspecto festoneado de la mucosa $(6,24)$. Los cambios en el íleon terminal, el ciego y el colon ascendente, pueden revelar estrechez, obstrucción, presencia de masa o distensión que sugieren tumor.

La anatomía patológica es el examen definitivo para confirmar el diagnóstico de la angiostrongilosis abdominal. La mayoría de los estudios han sido encabezados por un patólogo o han tenido la participación de éste. R. Céspedes era un patólogo. La frecuente presencia de casos en Brasil llevó al establecimiento de criterios de diagnóstico anatomopatológico de la enfermedad, macro y microscópicos, así: 1) macroscópicos: engrosamiento de la pared, con patrón pseudotumoral y lesión necrótica congestiva; 2) microscópicos: infiltración masiva de eosinófilos en toda las capas, granulomas y vasculitis eosinofílica de todo tipo de vasos (26).

Los especímenes quirúrgicos usualmente incluyen longitudes diversas del íleon distal, el apéndice, el ciego y el colon ascendente. El área lesionada aparece edematosa o rígida y característicamente engrosada, con aumento de su espesor $4-5$ veces lo normal. El engrosamiento puede ser concéntrico o segmentario y reduce notoriamente la luz, como se ve en las figuras 1-2, hecho que sugiere aspecto tumoral. Pueden apreciarse gránulos amarillentos subserosos que representan granulomas parasitarios. Al corte, la masa engrosada es homogénea y amarillenta; puede haber úlceras intestinales puntiformes o extensas, estenosis y perforación. En el mesenterio, hay adenopatías individuales de $2-3 \mathrm{~cm}$, firmes, que en el examen microscópico pueden tener granulomas y eosinofilia con presencia de huevos y larvas del parásito.

Microscópicamente, el engrosamiento de la pared intestinal se debe a infiltrado inflamatorio difuso, granulomatoso, con algunos focos de necrosis, que va desde la mucosa a la serosa y es particularmente rico en eosinófilos, con presencia de cristales de Charcot-Leyden. Estos se ven mejor con la coloración de Zielh-Neelsen o de Fite-Faraco (27). Su búsqueda en el examen coprológico podría ser una ayuda diagnóstica importante ante un caso sospechoso.

En la paciente aquí presentada, las larvas y huevos fueron muy poco abundantes en la lámina propia de la mucosa, en la muscular y en la serosa, aunque en estas capas estaba presente el infiltrado inflamatorio difuso rico en eosinófilos. En la submucosa ocurrió el mayor engrosamiento con presencia de enorme número de huevos, larvas y algunos parásitos dentro de las arterias.

En relación con esta distribución del parásito, que tiene importancia si se estudian biopsias del coIon, las cuales sólo incluyen la mucosa, hemos encontrado tan sólo una observación en la cual múltiples biopsias de la mucosa del colon transverso distal permitieron hacer el diagnóstico de angiostrongilosis abdominal (9).

Otro hallazgo esencial es el daño vascular de arterias submucosas pequeñas en donde con 
mayor frecuencia se encuentran los parásitos adultos que pueden llegar a ocluir la luz vascular. También se pueden comprometer las venas, los capilares y los linfáticos (26). Las arterias presentan proliferación de la íntima, ruptura de la lámina elástica interna, hipertrofia de la capa muscular e infiltración de su pared por granulomas y eosinófilos, que atacan al parásito o a sus huevos en un patrón denominado vasculitis eosinofílica $(15,26)$ característico de la enfermedad, muy útil cuando no se encuentran los parásitos, sus huevos o larvas, para sospechar la existencia de la angiostrongilosis (26). La trombosis de estos vasos o su ruptura origina infartos intestinales o sangrado, como ocurrió en la niña presentada.

Las larvas y el parásito se han encontrado también en los ganglios linfáticos regionales, en el hígado (28) y en las arterias espermáticas, localización en la cual causan necrosis testicular aguda $(6,29)$.

En resumen, el diagnóstico definitivo de la angiostrongilosis abdominal es anatomopatológico. La clínica es la de un cuadro abdominal agudo o subagudo que sugiere apendicitis. El colon por enema muestra defectos de llenado y festoneamiento de la mucosa, engrosamiento y estenosis intestinal. El cuadro hemático no muestra leucocitosis ni eosinofilia en 10 a $15 \%$ de los casos. Se sugiere investigar la presencia de grandes cantidades de cristales de Charcot Leyden en la materia fecal, como una ayuda ante un caso sospechoso, dada su abundancia en las lesiones histológicas. En Costa Rica y El Salvador (7-8, 24,30 ) se han diseñado pruebas inmunológicas para el diagnóstico de casos y para investigaciones epidemiológicas, usando antígenos purificados de larvas o de adultos obtenidos de la infección experimental en ratas, pruebas útiles que no están disponibles en Colombia.

Para el diagnóstico diferencial, se debe tener en cuenta que $A$. costaricensis es el único parásito que invade las arterias ileocecales y el único en el que se ven simultáneamente numerosos huevos y larvas del helminto es en los cortes histopatológicos (13). Strongyloides stercoralis predomina en el duodeno y en el píloro y no invade de manera masiva y severa todas las capas de la pared intestinal. En la forma diseminada grave con autoinfección interna, las larvas de Strongyloides pueden penetrar la pared del colon y como signo de mal pronóstico, no se acompañan de eosinofilia (31). La eosinofilia masiva de la pared intestinal es propia de la angiostrongilosis y no de la estrongiluidiasis. Los granulomas por huevos de ascaris pueden observarse en la pared intestinal pero suelen ser más frecuentes en el peritoneo. Los huevos tienen morfología característica, con cutícula gruesa, festoneada, mientras que los huevos de Angiostrongylus no demuestran una cubierta o cutícula, la cual es tan tenue que parece no existir. Schistosoma mansoni es el único parásito de este género en América que también vive en los vasos, pero éstos son ramas de la vena porta y los granulomas que originan sus huevos se localizan en el hígado. La esquistosomiasis no se ha descrito en Colombia. La localización errática ocasional de Enterobius vermicularis en la pared del intestino grueso o causando granulomas peritoneales, se identifica por el característico aspecto de los huevos (32).

En general, el tratamiento de la angiostrongilosis abdominal es quirúrgico. En algunas formas no muy severas de la enfermedad, se ha usado tiabendazol y dietilcarbamazina, medicamentos que también se indican en el postoperatorio de la enfermedad $(6,7,9)$. No sabemos si se ha usado ivermectina, el antiparasitario reciente de uso exitoso en muchas parasitosis humanas. El pronóstico en general es bueno; en Costa Rica han fallecido dos niños de 194 tratados en un período de 19 años (7), mientras que en Brasil, sobre menos de 50 casos había ya $7 \%$ de letalidad (17).

La prevención de esta enfermedad rural y suburbana incluye el control de las babosas que, junto con los roedores, huéspedes definitivos del parásito, constituyen una plaga de diferentes tipos de cultivos $V$. plebeius tiene hábitos nocturnos y en algunas zonas de Costa Rica se ha demostrado que hasta el $75 \%$ de estas babosas está infectadas por A. costaricensis (24). La viabilidad de las larvas en las heces de las ratas es de 8-10 días, mientras que en la baba excretada por el molusco viven hasta la desecación de este material (24).

\section{La angiostrongilosis en Colombia}

La enfermedad se reconoció por primera vez en 1979, cuando el patólogo de la Universidad del 
Valle, Carlos Cuello, presentó en el XXI Congreso de la Sociedad Colombiana de Patología el estudio de un niño procedente de Dagua, Valle, operado por un cuadro de apendicitis aguda, órgano en el cual pudo ver el parásito adulto en los vasos y sus larvas y huevos en la pared apendicular (33) (cuadro 1). El doctor Cuello había trabajado varios meses en Costa Rica con el doctor Céspedes y había visto y estudiado con él muchos casos de angiostrongilosis. El doctor Céspedes confirmó este diagnóstico.

El doctor E. A. Malek, parasitólogo de la Universidad de Tulane, visitante de la Universidad del Valle, estudió un área campesina de Dagua, vecina a la residencia del enfermo anterior y encontró lesiones intestinales por el parásito en 2 roedores, Oryzomys caliginous, pero no encontró larvas en varios moluscos. Logró la infección experimental del molusco Varonicella occidentalis (34).

El doctor Gonzalo Bermúdez, patólogo de Ibagué, estudió en 1983 a un hombre de 65 años, procedente de la desaparecida ciudad de Armero, Tolima, quien había sido intervenido por un cuadro abdominal agudo diagnosticado como apendicitis y que incluyó resección del íleon distal. Grandes masas de la pared engrosada contenían parásitos y huevos y algunos gusanos adultos en los vasos mesentéricos. En la consulta hecha al Laboratorio de Patología del Instituto Nacional de Salud, se diagnosticó angiostrongilosis abdominal.

Los patólogos del Hospital Militar Central de Bogotá, Anita Herrán y Gustavo Pérez, estudiaron el especimen quirúrgico de un niño de 5 años de edad, procedente de Puerto Leguízamo, Putumayo, con enfermedad de 20 días de evolución, con presencia de masa voluminosa en la fosa iliaca derecha, leucocitosis de $23.000 / \mathrm{mm}^{3}$ y eosinofilia de 14\%; el parásito se identificó en la luz de las arterias y las larvas en la pared intestinal (35).

En 1987, los patólogos del Hospital Militar Central estudiaron el especimen quirúrgico de un joven de 17 años de edad cuya procedencia desconocemos. La pieza anatómica comprendía $4 \mathrm{~cm}$ del íleon distal, el apéndice, el ciego y $15 \mathrm{~cm}$ del colon ascendente. Se demostró inflamación granulomatosa severa, ulceración y parasitismo masivo de la pared del ciego y del colon con ocasional presencia de parásitos intravasculares, con erosión y ruptura de una rama submucosa de la arteria apendículo-cólica. El parásito no se identificó en ese entonces, pero junto con el doctor Orlando Calderón, patólogo del Hospital Militar Central, concluimos ahora que se trataba de angiostrongilosis abdominal.

En 1993, se atendió en el Hospital San Juan de Dios de Bogotá a una mujer indígena de 34 años de edad, procedente de Mitú, Vaupés, remitida por presentar masa en la fosa iliaca derecha y hematoquecia. La notoria presencia de huevos y larvas en la pared intestinal se interpretó inicialmente como estrongiloidiasis, pero en el Instituto de Salud en el Trópico de la Universidad Nacional se confirmó como angiostrongilosis abdominal (25).

En conclusión, la angiostrongilosis abdominal es un parasitismo intestinal grave, potencialmente fatal y de tratamiento quirúrgico frecuente. Su presencia se ha demostrado en amplias áreas colombianas y aún cuando sólo se ha documentado media docena de casos humanos, su estudio se debe enfatizar en todas nuestras

Cuadro 1. Angiostrongilosis abdominal en Colombia.

\begin{tabular}{llll}
\hline Autor & Año & Caso & Procedencia \\
\hline C. Cuello (33) & 1979 & Niño de 5 años & Dagua, Valle \\
E.A. Malek (34) & 1981 & $\begin{array}{l}2 \text { de } 7 \text { Oryzomys caliginous } \\
\text { Varonicella occidentalis }\end{array}$ & $\begin{array}{l}\text { Dagua, Valle } \\
\text { Hombre, 65 años }\end{array}$ \\
G. Bermúdez & & Niño, 5 años & Armero, Tolima \\
A. Herrán, G. Pérez (35) & 1983 & Soldado, 17 años & Puerto Leguízamo, Putumayo \\
O. Calderón & 1986 & Mujer, 35 años & ? \\
A. Corredor y col. (25) & 1987 & Niña, 4 años & Mitú, Vaupés \\
G. Rodríguez & 1993 & Carurú, Vaupés \\
\hline
\end{tabular}


escuelas de medicina, pues es posible que el desconocimiento de la enfermedad entre los médicos sea una causa importante de su aparente baja frecuencia. En los patólogos recae el diagnóstico final de la enfermedad, mientras no dispongamos de las pruebas inmunológicas para realizarlo. Por otra parte, el envío de toda biopsia o pieza quirúrgica para estudio histológico es una conducta ética y una norma establecida en el Código Sanitario Nacional que evitará el subdiagnóstico de esta parasitosis.

\section{Agradecimientos}

Al doctor Orlando Calderón, patólogo del Hospital Mílitar de Bogotá, por permitirme examinar las láminas de los casos de angiostrongilosis de ese hospital; al doctor Augusto Corredor por permitirme ver los cortes histológicos y el trabajo de la otra paciente de Mitú; al doctor Santiago Nicholls por obsequiarme una copia de la película del doctor Morera sobre angiostrongilosis; al histotecnólogo Carlos Polanco por realizar innumerables cortes de buena calidad, hasta encontrar el parásito dentro de los vasos y al doctor Carlos Eduardo Pérez por su ayuda en la búsqueda de referencias.

\section{Referencias}

1. Rodríguez G, Sarmiento L, Silva A. Pénfigo foliáceo endémico en indígenas colombianos. Rev Soc Col Dermatol 1993;2:91-4.

2. Rodríguez G. Lobomycosis. Int J Dermatol 1993;32:324-32.

3. Rodríguez G, Téllez N. Lobomycosis in Colombian Amerindian patients. Mycopathologia 1992;120:5-9.

4. Morera P, Céspedes R. Angiostrongilosis abdominal: una nueva parasitosis humana. Acta Med Costarric 1971;14:159-73

5. Céspedes R, Salas J, Mekbel S, Troper L, Mulhner F, Morera P. Granulomas entéricos y linfáticos con intensa eosinofilia tisular producidos por un estrongilideo (Strongylata). Acta Med Costarric 1967;10:235-55.

6. Loria-Cortes R, Lobo-Sanahuja JF. Clinical abdominal angiostrongylosis. A study of 116 children with intestinal eosinophilic granuloma caused by Angiostrongylus costaricensis. Am J Trop Med Hyg 1980;29:538-44

7. Lobo-Sanahuja JF, Loria-Cortes R, González G. Angiostrongilosis abdominal: aspectos clinicos, tratamiento y revisión de la literatura. Bol Med Hosp Infant Mex 1987;44:4-9.
8. Morera P. Abdominal angiostrongylosis: a problem of public health. Parasitol Today 1985;1:173-5.

9. Juminer B, Roudier M, Raccurt CP, Pujol HP, Gerry F, Bonnet R. Presence of angiostrongylose abdominale en Guadeloupe. A propos de deux cas récents. Bull Soc Path Exo 1992;85:39-43

10. Kevin-Baird J, Neafie RC, Lanoie L, Connor DA. Abdominal angiostrongylosis in an African man: case study. Am J Trop Med Hyg 1987;37:353-6

11. Ubelaker JE, Hall M. First report of Angiostrongylus costaricensis Morera and Cèspedes 1971 in the Unites States. J parasitol 1979;65:307-10.

12. Silvera TC, Ghali VS, Roven S, Heimann J, Gelb A. Angiostrongyliasis: a rare cause of gastrointestinal hemorrhage. Am J Gastroenterol 1989;84:329-32.

13. Neafie RC, Marty AM. Unusual infectiuon in humans. Clin Microbiol Rev 1993;6:34-56.

14. Hulbert TF, Larsen RA. Abdominal angiostrongyliasis mimicking acute appendicitis and Meckel's diverticulum: report of a case in the United States and review. Clin Infect Dis 1992;14:836-40.

15. Agostini AA, Marcolan AM, Lisot JM, Lisot JUF. Angiostrongyliase abdominal. Estudio anátomopatológico de quatro casos observados no Rio Grande do Sul, Brasil. Mem Inst O Cruz 1984;79:443-5.

16. Ayala RMA. Angiostrongiloidiase abdominal. Seis casos observados no Paranà e em Santa Catarina, Brasil. Mem Inst O Cruz 1987;82:29-36.

17. Graeff-Tixeira C, Camillo-Coura L, Lenzi HL. Clinical and epidemiological aspects of abdominal angiostrongyliasis in Southern Brazil. Rev Inst Med Trop Sao Paulo 1991;33:373-8.

18. Pena MG, Andrade Filho JS, Assis SC. Angiostrongylus costaricensis: first record of its occurence in the State of Espirito Santo, Brazil, and a review of its geographic distribution. Rev Inst Med Trop Sao Paulo 1995;37:369-74

19. Morera P. Life history and redescription of Angiostrongylus costaricensis Morera and Céspedes, 1971. Am J Trop Med Hyg 1973;22:613-21.

20. Ubelaker JE. Systematics of species referred to the genus Angiostrongylus. J Parasitol 1986;72:237-44.

21. Portes Santos C. Redescricao de Angiostrongylus (Parastrongylus) costaricensis isolado de novo hospedeiro silvestre, Proechimys sp., na Venezuela (Metastrongyloidea, Angiostrongylidae). Mem Inst O Cruz 1985;80:81-3.

22. Kevin-Baird J. Abdominal parastrongylosis. En: Connor $\mathrm{DH}$, Chandler FW, et al., editors. Pathology of infectious diseases. First edition. Stanford: Appleton \& Lange; 1997. p 1531-5.

23. Tesh RB, Ackerman LJ, Dietz WH, Williams JA. Angiostrongylus costaricensis in Panamá. Prevalence 
and pathologic findings in wild rodents infected with the parasite. Am J Trop Med Hyg 1973;22:348-56.

24. Morera P, Conejo M, Castro A. Angiostrongiliasis abdominal: un problema de salud pública. Película dirigida por Fernando Armati, realizada por el Instituto Italo Latinoamericano (IILA), Studio di cinematografía científica, Roma. San José: Universidad de Costa Rica; 1995.

25. Corredor A, Echeverry MC, Salcedo H, Alvarado $\mathrm{H}$, Méndez E. Intestinal hemorrhage caused by Angiostrongylus costaricensis: a case in Vaupés, Colombia. (sometido para publicación).

26. Graeff-Teixeira C, Camillo-Coura L, Lenzi HL. Histopathological criteria for the diagnosis of abdominal angiostrongyliasis. Parasitol Res 1991;77:606-11.

27. Rodríguez RA, Sarmiento L, Rodríguez G. Los cristales de Charcot-Leyden. Biomédica 1998;18:8992.

28. Morera P, Pérez F, Mora F, Castro L. Visceral larva migrans-like syndrome caused by Angiostrongylus costaricensis. Am J Trop Med Hyg 1982;31:67-70.
29. Ruiz PJ, Morera P. Spermatic artery obstruction caused by Angiostrongylus costaricensis Morera y Céspedes 1971. Am J Trop Med Hyg 1983;32:1458-9.

30. Sauerbrey M. A precipitin test for the diagnosis of human abdominal angiostrongyliasis. Am J Trop Med Hyg 1977;26:1156-8.

31. Panqueva C, Rodríguez G, Téllez N. Estrongiloidiasis diseminada. Biomédica 1986;6:115-26.

32. Chandrasoma PT, Mendis NK. Enterobius vermicularis in ectopic sites. Am J Trop Med Hyg 1977; 26:644-9.

33. Cuello C. Angiostrongiloidiasis abdominal. Primer caso en Colombia. Memorias, XXI Congreso Colombiano de Patología, Cartagena; 1979.

34. Malek EA. Presence of Angiostrogylus costaricensis Morera y Céspedes in Colombia. Am J Trop Med Hyg 1981;30:81-3.

35. Herrán A, Pérez G. Angiostrongyloidiasis intestinal. Hosmil Médica 1986;7:43-4. 